\title{
High immunoexpression of Ki67, EZH2, and SMYD3 in diagnostic prostate biopsies independently predicts outcome in patients with prostate cancer
}

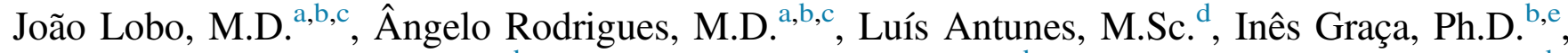 \\ João Ramalho-Carvalho, B.Sc. ${ }^{\mathrm{b}}$, Filipa Quintela Vieira, Ph.D. ${ }^{\mathrm{b}, \mathrm{e}}$, Ana Teresa Martins, M.Sc. ${ }^{\mathrm{a}, \mathrm{b}}$, \\ Jorge Oliveira, M.D. , Carmen Jerónimo, Ph.D. ${ }^{\mathrm{b}, \mathrm{c}, 1}$, Rui Henrique, M.D., Ph.D. ${ }^{\mathrm{a}, \mathrm{b}, \mathrm{c}, *, 1}$ \\ Department of Pathology, Portuguese Oncology Institute of Porto, Porto, Portugal \\ ${ }^{\mathrm{b}}$ Cancer Biology and Epigenetics Group, Research Center (CI-IPOP), Portuguese Oncology Institute of Porto, Porto, Portugal \\ ${ }^{c}$ Department of Pathology and Molecular Immunology, Institute of Biomedical Sciences Abel Salazar (ICBAS), University of Porto, Porto, Portugal \\ ${ }^{\mathrm{d}}$ Department of Epidemiology, Portuguese Oncology Institute of Porto, Porto, Portugal \\ ${ }^{\mathrm{e}}$ School of Allied Health Sciences (ESTSP), Polytechnic of Porto, Vila Nova de Gaia, Portugal \\ ${ }^{\mathrm{f}}$ Department of Urology, Portuguese Oncology Institute of Porto, Porto, Portugal \\ ${ }^{1}$ Joint senior authors. \\ E-mail addresses: rmhenrique@icbas.up.pt, henrique@ipoporto.min- \\ saude.pt (R. Henrique).
}

\begin{abstract}
Introduction: Overtreatment is a major concern in patients with prostate cancer (PCa). Prognostic biomarkers discriminating indolent from aggressive disease in prostate biopsy are urgently needed. We aimed to evaluate the prognostic value of Ki67, EZH2, LSD1, and SMYD3 immunoexpression in diagnostic biopsies from a cohort of PCa patients with long term follow-up.

Materials and methods: A series of 189 consecutive prostate biopsies diagnosed with PCa (1997-2001) in a cancer center was included in the study, with follow-up last updated in November 2016. Biopsies were reviewed and graded according to 2016 WHO criteria. Immunohistochemistry was performed in the most representative block. Nuclear staining was assessed using digital image analysis. Study outcomes included disease-specific, disease-free, and progression-free survival. Statistical analysis was tabulated using SPSS version 22.0. Survival curves and hazard ratios (HRs) were estimated using Kaplan-Meyer and Cox-regression models, respectively. Statistical significance was set at $P<0.05$.

Results: The proportion of patients who completed the study was 177/189 (94\%). In univariable analysis, high Ki67, EZH2, and SMYD3 immunoexpression associated with significantly worse disease-specific survival (HR $=1.86,95 \% \mathrm{CI}: 1.05-3.29$; HR $=1.87,95 \% \mathrm{CI}$ : 1.10-3.27; HR = 2.68, 95\% CI: 1.02-7.92). In multivariable analysis, the 3 biomarkers displayed significantly worse DSS adjusted for CAPRA score $(\mathrm{HR}=1.78,95 \% \mathrm{CI}: 1.01-3.16 ; \mathrm{HR}=1.93,95 \% \mathrm{CI}: 1.12-3.32 ; \mathrm{HR}=2.71,95 \%$ CI: 1.04-7.10). Among patients with low/intermediate risk CAPRA score, high Ki67 immunoexpression identified those more prone to experience disease recurrence $(\mathrm{HR}=$ 9.20, 95\% CI: $1.27-66.44)$ and progression (HR $=2.97,95 \%$ CI: $1.05-8.43)$.

Conclusions: High Ki67, EZH2, and SMYD3 immunoexpression, adjusted for standard clinicopathological parameters, independently predicts outcome in patients with $\mathrm{PCa}$, at diagnosis. This might assist in discriminating indolent from aggressive PCa, improving treatment selection.

Keywords: Biopsy; EZH2; Ki67; Prognosis; Prostate cancer; SMYD3

This work was supported by the Research Center of Portuguese Oncology Institute of Porto (Grant nos. FB-GEBC-27 and 19-CI-IPOP2016) and the European Community's Seventh Framework Programme (Grant no. FP7-HEALTH-F5-2009-241783). J.R.-C. and I.G. are supported by FCT- Fundação para a Ciência e Tecnologia, Portugal fellowships (Grant nos. SFRH/BD/71293/2010 and POCI-01-0145-FEDER-006868, respectively).
\end{abstract}




\section{Introduction}

Prostate cancer $(\mathrm{PCa})$ is the most prevalent and the second most common malignant neoplasm in men, comprising $15 \%$ of cancers diagnosed worldwide in 2012, with $75 \%$ occurring in industrialized countries. It constitutes the fifth cause of cancer-related death in males, accounting for $6.6 \%$ of the total. More than 1.8 million new cases are expected in 2030, about 758,000 more than in 2012 [1]. In the United States, it is estimated that $12.9 \%$ of men will be diagnosed $\mathrm{PCa}$ during their lifetime [2,3].

$\mathrm{PCa}$ diagnosis generally requires histological examination of prostate biopsy cores, allowing for evaluation of parameters that predict disease aggressiveness, including grading. Gleason score (GS) has been the mainstay of $\mathrm{PCa}$ grading for over 4 decades [4], despite subsequent refinements [5] and introduction of grade groups (GG) [6], recently adopted by the newest WHO (World Health Organization) classification [7]. Its ability to prognostically stratify patients with $\mathrm{PCa}$ was validated in large cohorts, both in radical prostatectomy (RP) and prostate biopsy specimens, considering relapse-free [8] or disease-specific [9] survival (DSS) as endpoints.

Owing to disseminated serum prostate-specific antigen (PSA) screening, $80 \%$ of patients with $\mathrm{PCa}$ are diagnosed with organ-confined disease, entailing excellent 5-year survival [3]. Given the 5-fold difference between incidence and mortality, choosing optimal treatment and avoiding overtreatment constitutes a major challenge. Standard clinicopathological parameters, however, cannot accurately differentiate indolent from aggressive disease and, thus, discovery and validation of biomarkers that better predict disease behavior and assist clinicians in treatment tailoring, is imperative. Notwithstanding, most biomarkers reported have been difficult to validate, with most studies focusing on RP specimens and very few analyzing cohorts of patients managed conservatively [10-12]. As diagnostic biopsies represent the whole spectrum of PCa and allow for assessment before therapeutic decision, validation of biomarkers that may accurately distinguish clinically insignificant from curable and from lethal disease is mandatory.

Although molecular pathology has thrived in recent years, immunohistochemistry remains a major ancillary tool in pathology owing to its widespread availability. Immunohistochemistry-based biomarkers offer a significant advantage for universal, fast and reliable transference to clinical routine. Despite the plethora of reported immunohistochemical biomarkers with potential for PCa prognostication, however, none has reached clinical use, mostly owing to lack of reproducibility of evaluation procedures [10-14]. Herein, using a cohort of pretherapeutic $\mathrm{PCa}$ biopsies, we sought to evaluate the prognostic value of 4 biomarkers previously shown to have clinical potential, including Ki67 [10-14], and a panel of epigenetic modifiers-EZH2, LSD1, and SMYD3 - that we and others have shown to convey prognostic information and allow for discrimination of aggressive $\mathrm{PCa}$ among patients submitted to RP [15-21]. To increase the accuracy and consistency of results, evaluation of nuclear biomarkers was performed using a digital image analysis system, and cutoffs were determined based on data distribution.

\section{Materials and methods}

\subsection{Patients and samples}

Prostate biopsies from patients consecutively diagnosed with PCa at Portuguese Oncology Institute of Porto (IPO Porto, a tertiary healthcare institution) from 1997 to 2001 were enrolled after informed consent. All slides were reviewed by 2 uropathology dedicated pathologists and graded according to 2016 WHO criteria. Percentage of tumor in biopsy and number of involved cores were also recorded. A representative block (the one representing the highest GS) was selected for immunohistochemistry. When several cores in different blocks depicted the same prognostic GS, the one with greatest amount of tumor was selected for analysis.

Clinical data was retrieved from patients' charts (dates of birth, diagnosis, treatment start, biochemical recurrence (BCR), and death; serum PSA at diagnosis; clinical stage (CS); treatments performed; evidence of BCR or clinical recurrence/progression; last follow-up date; and vital status). Follow-up was updated as of 30th November, 2016. CAPRA score was calculated for each patient as a tool for risk assessment [22]. BCR/progression was considered as previously defined [23]. Patient death was rendered as $\mathrm{PCa}-$ related (death from disease) when patients died owing to progression/metastasized disease or in the sequence of treatments performed, or as $\mathrm{PCa}$-unrelated (including either death with disease or death with no evidence of disease).

\subsection{Immunohistochemistry and image analysis}

Immunohistochemistry was performed using Novolink Max Polymer Detection System (Leica Biosystems, Germany). Sections $(3 \mu \mathrm{m}$-thick) were cut and microwaved for 20 to 30 minutes in EDTA buffer at $800 \mathrm{~W}$ for antigen retrieval. 
Endogenous peroxidase was blocked through incubation in hydrogen peroxide in 3\% methanol for 30 minutes. Primary monoclonal antibodies for Ki67 (MIB-1, DAKO, Denmark), EZH2 (clone 6A10, Novocastra, UK), LSD1 (clone 1b2f2, Novus Biologicals, UK), and SMYD3 (Active Motif, Carlsbad, CA) were used at 1:100, 1:1,000, 1:1,750, and 1:300 dilution in $1 \%$ PBS-BSA, respectively, and incubated overnight at $4^{\circ} \mathrm{C}$. 3,3'-diaminobenzidine (Sigma-Aldrich, Germany) was used for visualization and hematoxilin for nuclear counterstaining. Appropriate positive controls were used for each antibody and negative control consisted on omission of primary antibodies. Because all cases analyzed contained nonneoplastic prostatic epithelium, this served as (internal) negative control as appropriate. However, when the immunohistochemical protocols were developed, negative external controls were used.

SMYD3 immunoexpression was assessed as previously described [15,16]. Digital image analysis system (GenASIs, Israel) was used for $\mathrm{Ki} 67, \mathrm{EZH} 2$, and LSD1 nuclear immunostaining quantification. Nuclei were considered positive if any staining was present, independently of intensity. Study protocol is outlined in detail in Fig. 1.

\subsection{Statistical analysis}

For analysis purposes, patients were divided into $3 \mathrm{CS}$ groups (stage I/II, stage III, and stage IV) and 2 major GG subgroups (GG 1-3 and 4-5). Patients were also divided into low/intermediate $(<6)$ and high risk $(\geq 6)$ CAPRA score. SMYD3 immunoexpression was categorized as weak, moderate, or strong. Cutoffs for Ki67, EZH2, and LSD1 nuclear immunostaining (high vs. low) were set at respective 75th percentile (P75). Diagnostic performance of other Ki67 cutoff values reported [12,24-32] was also assessed. Association between biomarkers' distribution and GG or CS was evaluated using chi-square test. Distribution of continuous variables between groups was compared using nonparametric tests (Mann-Whitney or Kruskall-Wallis, as appropriate). Survival curves were constructed using Kaplan-Meyer nonparametric estimator. Survival between groups was compared using log-rank test. Hazard ratios (HR) and respective 95\% CI were estimated using Cox-regression models. Statistical significance was set at $P<0.05$. Statistical analysis was performed using SPSS Statistics for Windows, version 22.0 (SPSS, Chicago, IL).

\section{Results}

\subsection{Cohort characterization and biomarker immunoexpression}

A total of 189 patients were enrolled and their clinicopathological features are depicted in Table 1. Most patients displayed high GS and GG, advanced disease stage, and high-risk CAPRA score.

Illustrative examples of Ki67, EZH2 and LSD1 (nuclear), and SMYD3 (cytoplasmic) immunostaining are shown in Fig. 2. Digital imaging-assisted immunoexpression assessment of nuclear biomarkers took approximately 5 minutes per case. The median number of tumor cells and of 200x fields evaluated per case, as well as respective P75

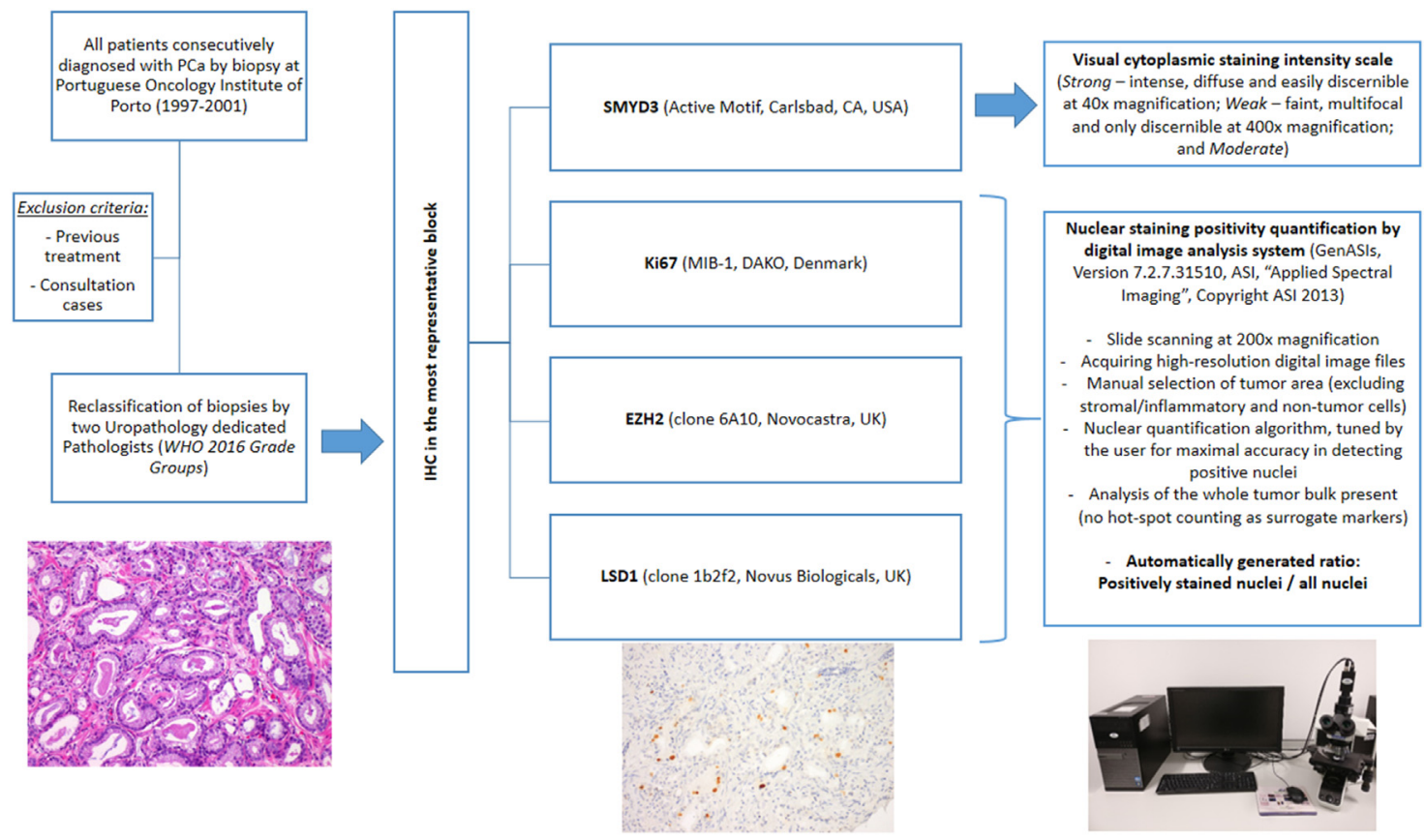

Fig. 1. Study methodology outline. EZH2 = enhancer of zeste homolog 2; IHC = immunohistochemistry; LSD1 = lysine-specific demethylase $1 ;$ PCa $=$ prostate cancer; SMYD3 = SET and MYND domain-containing protein 3; WHO = World Health Organization. 
Table 1

Clinicopathological features of patients with prostate cancer

\begin{tabular}{|c|c|}
\hline Age at diagnosis (y, median [IQR]) & $72(67-76)$ \\
\hline PSA at diagnosis (ng/ml, median [IQR]) & $43.0(15.8-124.0)$ \\
\hline \multicolumn{2}{|l|}{ Gleason grades, biopsy } \\
\hline $3+3$ & $25 / 189(13.2 \%)$ \\
\hline $3+4$ & $33 / 189(17.5 \%)$ \\
\hline $4+3$ & $45 / 189(23.8 \%)$ \\
\hline $4+4$ & $68 / 189(36.0 \%)$ \\
\hline $4+5$ & $14 / 189(7.4 \%)$ \\
\hline $5+4$ & $3 / 189(1.6 \%)$ \\
\hline $5+5$ & $1 / 189(0.5 \%)$ \\
\hline \multicolumn{2}{|l|}{ Prognostic grade groups, biopsy } \\
\hline Group 1 & $25 / 189(13.2 \%)$ \\
\hline Group 2 & $33 / 189(17.5 \%)$ \\
\hline Group 3 & $45 / 189(23.8 \%)$ \\
\hline Group 4 & $68 / 189(36.0 \%)$ \\
\hline Group 5 & $18 / 189(9.5 \%)$ \\
\hline \multicolumn{2}{|l|}{ Percentage of tumor, biopsy } \\
\hline$<25 \%$ & $52 / 189(27.5 \%)$ \\
\hline$<50 \%$ & $40 / 189(21.2 \%)$ \\
\hline$<75 \%$ & $59 / 189(31.2 \%)$ \\
\hline$\geq 75 \%$ & $38 / 189(20.1 \%)$ \\
\hline \multicolumn{2}{|l|}{ Clinical stage } \\
\hline Stage I & $3 / 188(1.6 \%)$ \\
\hline Stage II & $58 / 188(30.8 \%)$ \\
\hline Stage III & $71 / 188(37.8 \%)$ \\
\hline Stage IV & $56 / 188(29.8 \%)$ \\
\hline \multicolumn{2}{|l|}{ CAPRA score groups } \\
\hline <6 (low/intermediate risk) & $45 / 189(23.8 \%)$ \\
\hline$\geq 6$ (high risk) & $144 / 189(76.2 \%)$ \\
\hline \multicolumn{2}{|l|}{ Primary treatment } \\
\hline $\mathrm{RP} \pm$ others & $16 / 187(8.6 \%)$ \\
\hline RT alone or with ADT & $45 / 187(24.0 \%)$ \\
\hline ADT only & $123 / 187(65.8 \%)$ \\
\hline WW & $3 / 187(1.6 \%)$ \\
\hline \multicolumn{2}{|l|}{ Ki67 } \\
\hline P75 (cutoff value) & $5.33 \%$ \\
\hline Tumor cell count/case (median [IQR]) & $2,630(1,198-5,124)$ \\
\hline 200x fields/case (median [IQR]) & $8(4-11)$ \\
\hline \multicolumn{2}{|l|}{$\mathrm{EZH} 2$} \\
\hline P75 (cutoff value) & $12.40 \%$ \\
\hline Tumor cell count/case (median [IQR]) & $2,856(1,195-5,346)$ \\
\hline 200x fields/case (median [IQR]) & $9(5-12)$ \\
\hline \multicolumn{2}{|l|}{ LSD1 } \\
\hline P75 (cutoff value) & $41.40 \%$ \\
\hline Tumor cell count/case (median [IQR]) & $3,160(1,204-5,260)$ \\
\hline 200x fields/case (median [IQR]) & $8(5-12)$ \\
\hline \multicolumn{2}{|l|}{ SMYD3 } \\
\hline Weak & $25 / 178(14.0 \%)$ \\
\hline Moderate & $95 / 178(53.4 \%)$ \\
\hline Strong & $58 / 178(32.6 \%)$ \\
\hline
\end{tabular}

$\mathrm{IQR}=$ interquartile range; $\mathrm{P} 75=75$ th percentile for the percentage of positively stained nuclei; $\mathrm{RT}=$ radiation therapy; $\mathrm{WW}=$ watchful waiting.

value for nuclear biomarkers are provided in Table 1. PCa cases displaying Ki67, EZH2, or LSD1 immunostaining above P75, as well as those with strong SMYD3 cytoplasmic immunoexpression were designated "Ki67-high," "EZH2-high," "LSD1-high," and "SMYD3-high," respectively, for subsequent analyses.

Patients' age associated with GG $(P=0.003)$, and serum PSA at diagnosis with both GG and CS $(P<0.001$ for both) (Table 2). Significant association between Ki67, EZH2, LSD1, and SMYD3 immunoexpression, and GG, but not CS, was found $(P=0.001, P=0.003, P=$ 0.005 , and $P=0.004$, respectively). Serum PSA showed no association with Ki67, EZH2, LSD1, and SMYD3 immunoexpression $(P=0.750, P=0.184, P=0.837$, and $P=0.070)$.

\subsection{Survival analyses}

Complete follow-up data was available for 177/189 (94\%) patients and median follow-up time was 206 months (range: 0.6-232 mo). A total of $65(34.4 \%)$ patients died from $\mathrm{PCa}$ and 133 (70.4\%) displayed $\mathrm{BCR} /$ progression. Both GG (Fig. 3A) and CS (Fig. 3B) stratified patients in respect to DSS $(P<0.001$ for both).

\subsubsection{Univariable analysis}

3.2.1.1. Considering the whole cohort. Patients with SMYD3 strong and moderate immunoexpression experienced significantly worse overall survival (OS) compared to patients with weak expression (strong: HR $=2.19,95 \%$ CI: 1.21-3.97; moderate: $\mathrm{HR}=1.91,95 \%$ CI: 1.08-3.39).

Also, Ki67- and EZH2-high patients with PCa experienced significantly worse DSS (Ki67: HR $=1.86,95 \%$ CI: 1.05-3.29; EZH2: HR $=1.87,95 \%$ CI: $1.10-3.27$ ). No significant effect on DSS was found for LSD1 immunoexpression $(\mathrm{HR}=0.94,95 \% \mathrm{CI}: 0.50-1.75)$. SMYD3-high cases were also more likely to die from PCa compared to weak immunostaining ( $\mathrm{HR}=2.68,95 \% \mathrm{CI}$ : 1.02-7.92). Such association was not found for moderate vs. weak immunostaining (HR $=1.97,95 \%$ CI: 0.76-5.08) (Fig. 4).

Considering patients treated with curative intent (RP or radiation therapy), DSS, and OS analyses are not possible owing to the limited number of events.

3.2.1.2. Considering only androgen deprivation therapy treated patients. Ki67-high patients experienced significantly worse DSS (Ki67: HR = 1.91, 95\% CI: 1.05-3.47), but not those with EZH2-high (HR $=1.45$, 95\% CI: 0.832.55), LSD1-high (HR $=0.67,95 \%$ CI: 0.35-1.32), or SMYD3 strong immunoexpression $(\mathrm{HR}=1.57,95 \% \mathrm{CI}$ : $0.54-4.55)$.

\subsubsection{Nonstratified multivariable analysis}

3.2.2.1. Considering the whole cohort. Patients with SMYD3 strong and moderate immunoexpression had significantly worse OS adjusted for CAPRA score (strong: HR $=2.22,95 \%$ CI: $1.22-4.02$; moderate: $\mathrm{HR}=1.90,95 \%$ CI: $1.07-3.37)$.

Ki67-high, but not EZH2-high or SMYD3-high, patients had a significantly worse DSS adjusted for patient age, CS and GG (Ki67: HR = 1.91, 95\% CI: 1.01-3.57; EZH2: HR $=1.01,95 \%$ CI: 0.56-1.80; SMYD3: HR $=0.92,95 \%$ CI: 0.54-1.57). However, both Ki67 and EZH2-high cases displayed significantly worse DSS adjusted for CAPRA 

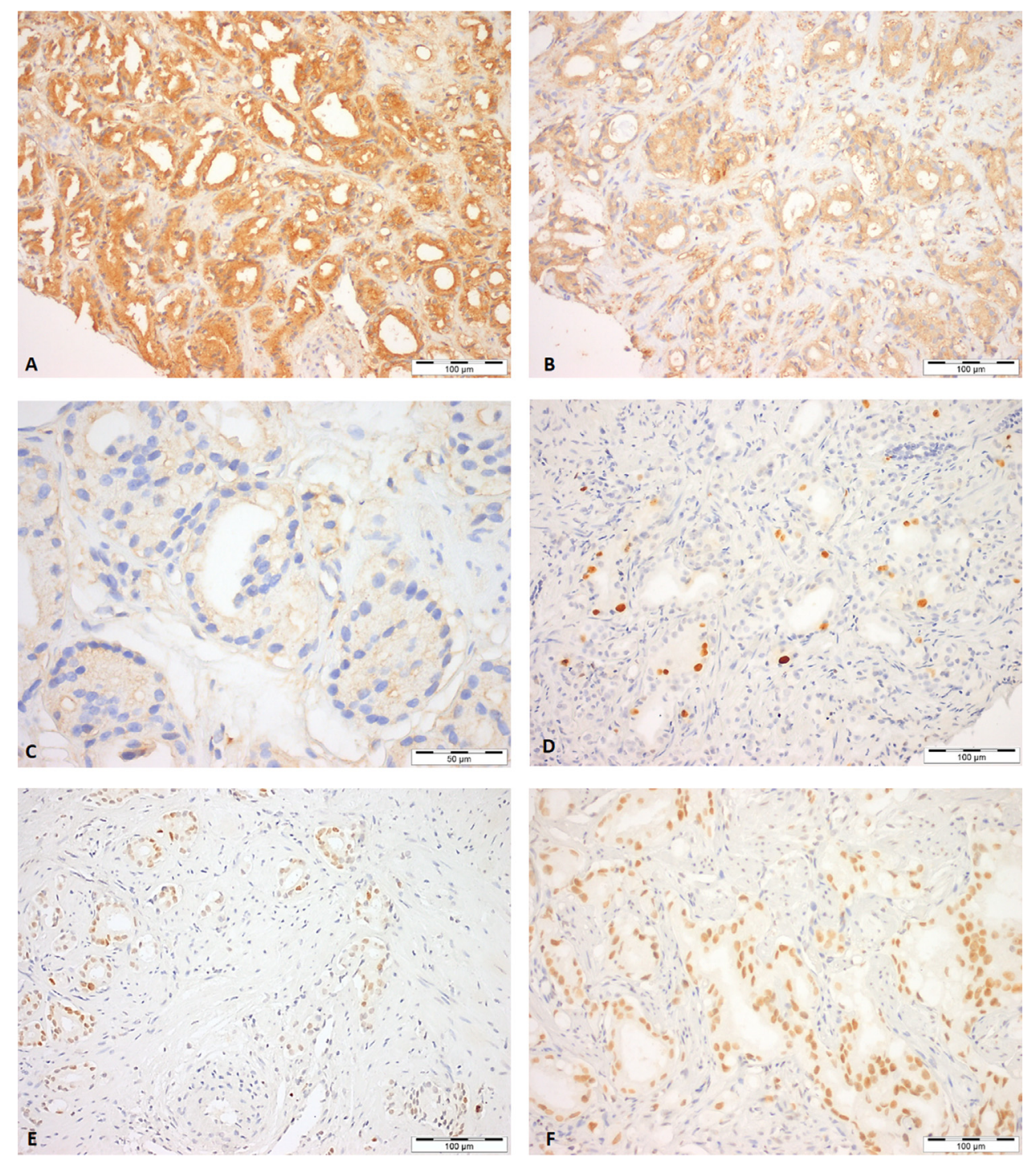

Fig. 2. Immunohistochemical expression of biomarkers in prostate cancer biopsies. (A-C) SMYD3 cytoplasmic staining (A) Strong intensity staining $(\times 200)$; and (B) moderate intensity staining $(\times 200)$; and $(C)$ weak intensity staining $(\times 400)$. (D) Ki67 nuclear staining $(\times 200)$; (E) EZH2 nuclear staining $(\times 200)$; (F) LSD1 nuclear staining $(\times 200)$.

score $(\mathrm{Ki} 67: \mathrm{HR}=1.78,95 \% \mathrm{CI}: 1.01-3.16 ; \mathrm{EZH} 2: \mathrm{HR}$ $=1.93,95 \%$ CI: 1.12-3.32). The same was apparent for SMYD3-high patients compared to weak SMYD3 immunostaining, adjusted for CAPRA score (HR $=2.71,95 \%$ CI: $1.04-7.10)$.

3.2.2.2. Considering only androgen deprivation therapy treated patients. Ki67-high patients experienced significantly worse DSS when adjusting for patient age, CS and GG (HR $=2.27,95 \%$ CI: 1.17-4.39), which did not occur for the other biomarkers $(\mathrm{EZH} 2: \mathrm{HR}=0.94,95 \% \mathrm{CI}$ : 0.52-1.72; SMYD3: HR $=0.82$, 95\% CI: $0.27-2.52$ ). When adjusting for CAPRA score patients with Ki67-high also showed significantly worse DSS (HR $=1.89,95 \% \mathrm{CI}$ : 1.04-3.45); the same was not disclosed for EZH2 or SMYD3-high cases (EZH2: HR $=1.46$, 95\% CI: 0.832.57; SMYD3: $\mathrm{HR}=1.59,0.55-4.60)$.

\subsubsection{Stratified multivariable analysis}

3.2.3.1. Considering the whole cohort. Among low/intermediate (but not high) risk CAPRA score, Ki67-high patients were significantly more likely to die from $\mathrm{PCa}(\mathrm{HR}=4.53$, 95\% CI: $1.36-15.10$ and $\mathrm{HR}=1.40,95 \%$ CI: $0.74-2.69$, respectively) (Table 3). Moreover, among GG 4 to 5, Ki67-high patients were significantly more likely to die from $\mathrm{PCa}$ when adjusted for patients' age and CS (HR $=2.18,95 \%$ CI: 1.09 4.38). Also, in GG 1 to 3 patients, EZH2-high cases displayed significantly worse DSS adjusted for patients' age and CS (HR $=3.66,95 \%$ CI: $1.15-11.60$ ).

3.2.3.2. Concerning only patients treated with curative intent ( $R P$ or radiation therapy). Low/intermediate (but not high) risk CAPRA score patients with Ki67-high were significantly more prone to experience disease recurrence 
Table 2

Association between clinical data and biomarker immunoexpression

\begin{tabular}{|c|c|c|c|c|}
\hline \multicolumn{5}{|l|}{ Grade groups } \\
\hline & Groups 1-3 & Groups 4-5 & & $P$ value \\
\hline Age (y, median [IQR]) & $71(66-75)$ & $74(70-78)$ & & 0.003 \\
\hline PSA (ng/ml, median [IQR]) & $21.7(11.6-64.5)$ & $93.2(38.6-253.9)$ & & $<0.001$ \\
\hline \multicolumn{5}{|l|}{ Biomarkers $(n, \%)$} \\
\hline Ki67 & & & & 0.001 \\
\hline$<$ P75 & $71 / 82(86.6 \%)$ & $48 / 76(63.2 \%)$ & & \\
\hline$\geq \mathrm{P} 75$ & $11 / 82(13.4 \%)$ & $28 / 76(36.8 \%)$ & & \\
\hline \multicolumn{4}{|l|}{ EZH2 } & 0.003 \\
\hline$<\mathrm{P} 75$ & $74 / 87(85.1 \%)$ & $50 / 78(64.1 \%)$ & & \\
\hline$\geq \mathrm{P} 75$ & $13 / 87(14.9 \%)$ & $28 / 78(35.9 \%)$ & & \\
\hline \multicolumn{4}{|l|}{ LSD1 } & 0.005 \\
\hline$<$ P75 & $70 / 82(85.4 \%)$ & $50 / 77(64.9 \%)$ & & \\
\hline$\geq \mathrm{P} 75$ & $12 / 82(14.6 \%)$ & $27 / 77(35.1 \%)$ & & \\
\hline \multicolumn{4}{|l|}{ SMYD3 } & 0.004 \\
\hline Weak & $20 / 100(20.0 \%)$ & $5 / 78(6.4 \%)$ & & \\
\hline Moderate & $56 / 100(56.0 \%)$ & $39 / 78(50.0 \%)$ & & \\
\hline Strong & $24 / 100(24.0 \%)$ & $34 / 78(43.6 \%)$ & & \\
\hline \multicolumn{5}{|l|}{ Clinical stage groups } \\
\hline & Stage I/II & Stage III & Stage IV & \\
\hline Age (y, median [IQR]) & $71(66-75)$ & $74(68-77)$ & $73(69-78)$ & 0.06 \\
\hline PSA (ng/ml, median [IQR]) & $14.0(8.8-23.2)$ & $43(21.2-89.0)$ & $204.0(93.5-953.0)$ & $<0.001$ \\
\hline \multicolumn{5}{|l|}{ Biomarkers $(n, \%)$} \\
\hline \multicolumn{4}{|l|}{ Ki67 } & 0.18 \\
\hline$<$ P75 & $43 / 51(84.3 \%)$ & $43 / 61(70.5 \%)$ & $32 / 45(71.1 \%)$ & \\
\hline$\geq \mathrm{P} 75$ & $8 / 51(15.7 \%)$ & $18 / 61(29.5 \%)$ & $13 / 45(28.9 \%)$ & \\
\hline \multicolumn{4}{|l|}{ EZH2 } & 0.12 \\
\hline$<$ P75 & $45 / 53(84.9 \%)$ & $45 / 63(71.4 \%)$ & $33 / 48(68.7 \%)$ & \\
\hline$\geq \mathrm{P} 75$ & $8 / 53(15.1 \%)$ & $18 / 63(28.6 \%)$ & $15 / 48(31.3 \%)$ & \\
\hline \multicolumn{4}{|l|}{ LSD1 } & 0.25 \\
\hline$<$ P75 & $41 / 51(80.4 \%)$ & $42 / 61(68.9 \%)$ & $37 / 46(80.4 \%)$ & \\
\hline$\geq \mathrm{P} 75$ & $10 / 51(19.6 \%)$ & $19 / 61(31.1 \%)$ & $9 / 46(19.6 \%)$ & \\
\hline \multicolumn{4}{|l|}{ SMYD3 } & 0.42 \\
\hline Weak & $9 / 57(15.8 \%)$ & $12 / 69(17.4 \%)$ & 4/51 (7.8\%) & \\
\hline Moderate & $33 / 57(57.9 \%)$ & $34 / 69(49.3 \%)$ & $27 / 51(53.0 \%)$ & \\
\hline Strong & $15 / 57(26.3 \%)$ & $23 / 69(33.3 \%)$ & $20 / 51(39.2 \%)$ & \\
\hline
\end{tabular}

$\mathrm{IQR}=$ interquartile range; $\mathrm{P} 75=75$ th percentile for the percentage of positively stained nuclei.
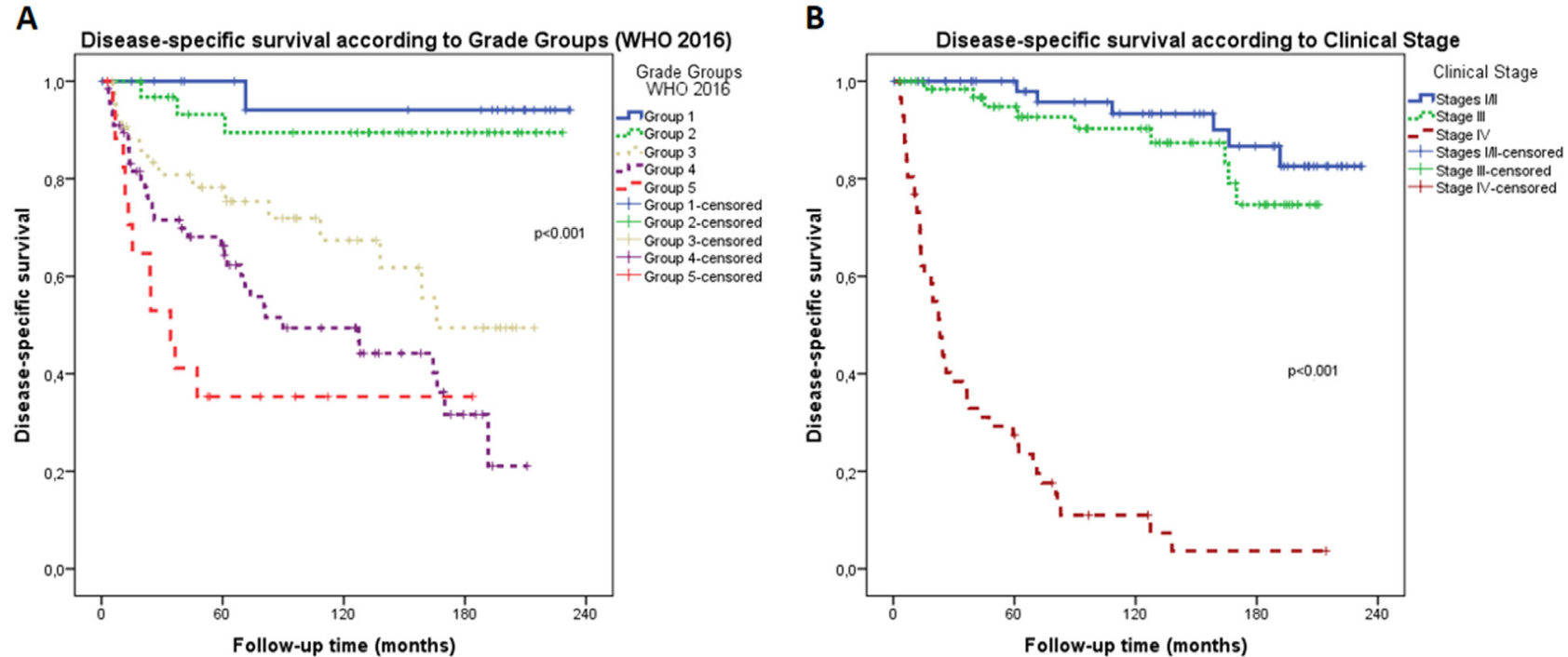

Fig. 3. Cohort stratification according to WHO grade groups and clinical stage, concerning disease-specific survival. (A) Disease-specific survival according to WHO grade groups. (B) Disease-specific survival according to clinical stage. WHO = World Health Organization. 
A

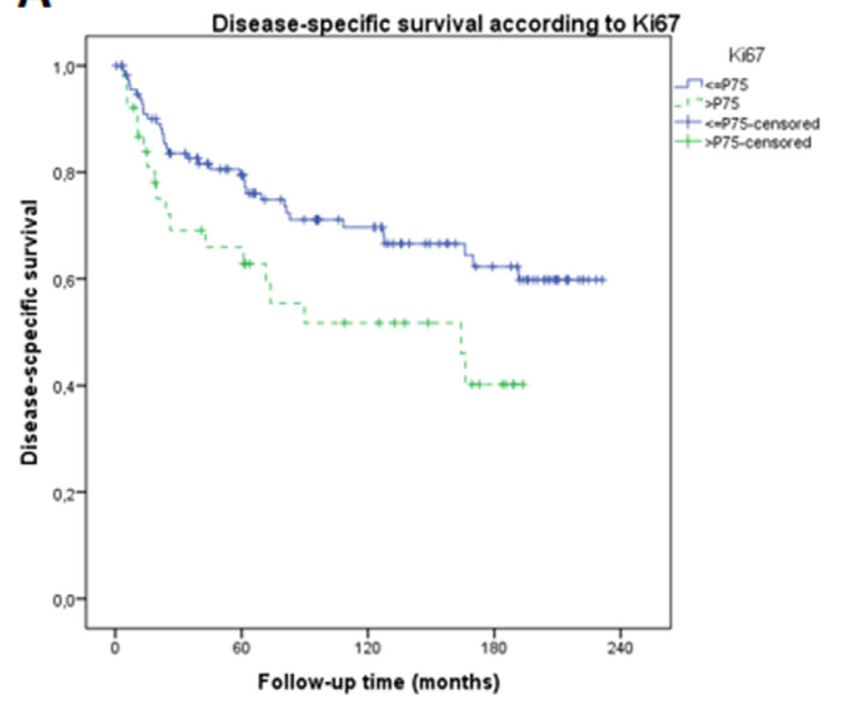

C

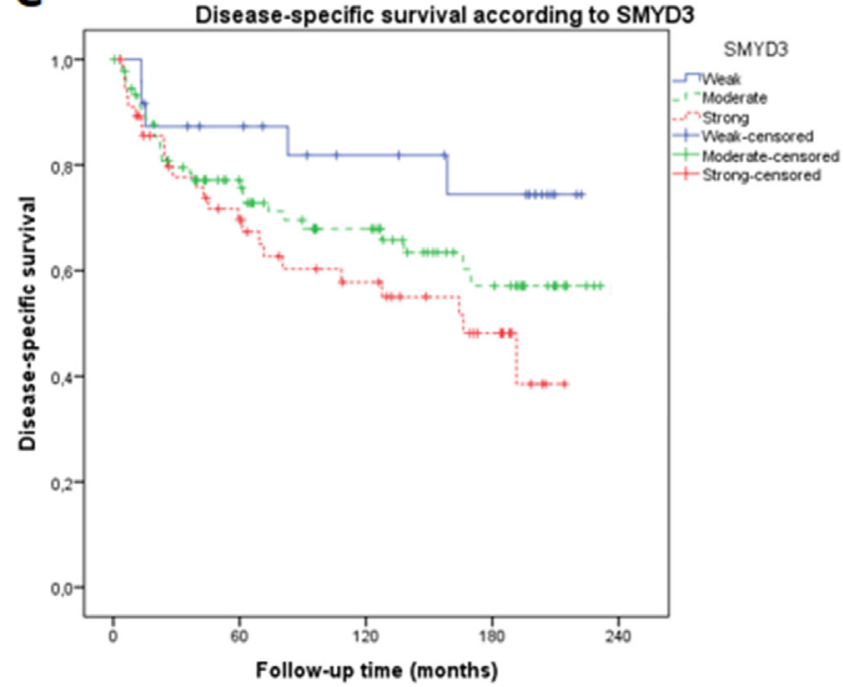

B

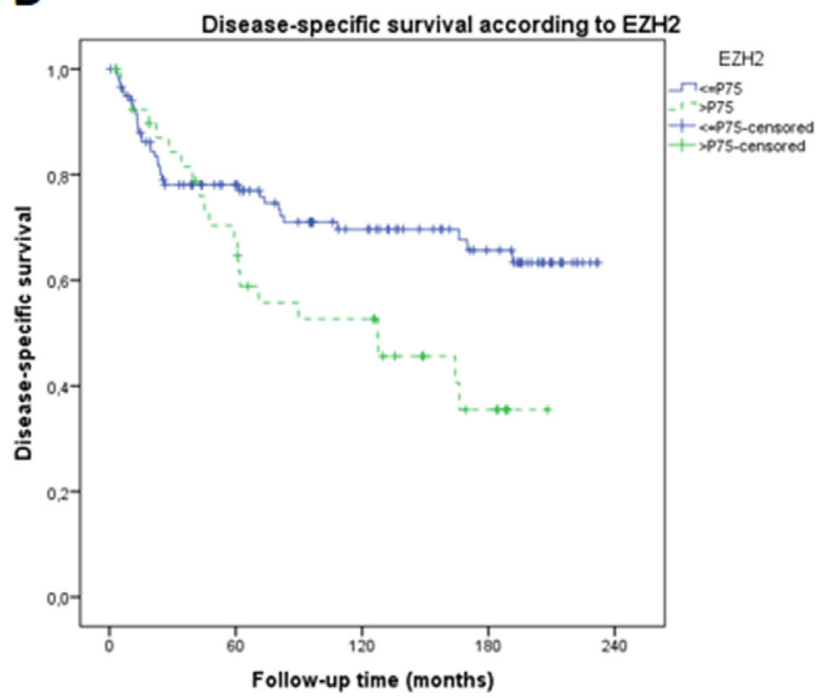

D

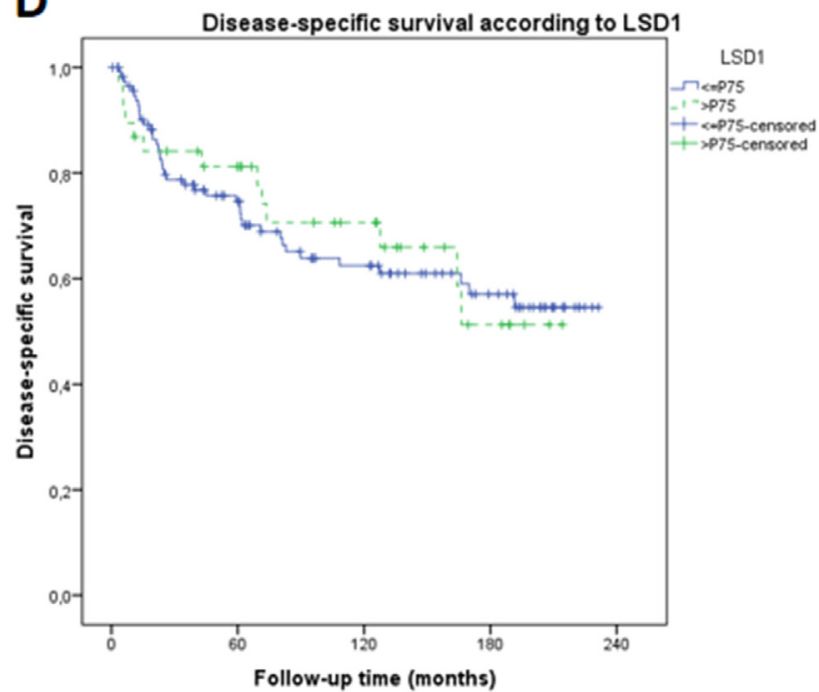

Fig. 4. Disease-specific survival according to biomarker expression levels: (A) Ki67, (B) EZH2, (C) SMYD3, and (D) LSD1.

$(\mathrm{HR}=9.20,95 \%$ CI: $1.27-66.44$ and $\mathrm{HR}=1.39,95 \%$ CI: $0.43-4.52$, respectively).

3.2.3.3. Concerning only androgen deprivation therapy treated patients. Low/intermediate (but not high) risk CAPRA score patients with Ki67-high were significantly more prone to experience disease progression $(\mathrm{HR}=2.97$, 95\% CI: $1.05-8.43$ and HR $=1.12,95 \%$ CI: $0.64-1.97$, respectively).

\subsection{Prognostic performance of Ki67 cutoffs reported in literature in this cohort}

The prognostic performance of other Ki67 cutoff values previously reported in literature was tested in our cohort (Table 4). Only $10 \%$ cutoff revealed a significant association with DSS both in univariable analysis $(\mathrm{HR}=2.32$,
95\% CI: 1.16-4.65) and adjusted for CAPRA score (HR = 2.25 , 95\% CI: $1.12-4.51$ ), whereas $5 \%$ cutoff only associated with DSS in univariable analysis.

\section{Discussion}

Growing evidence concerning PCa overdiagnosis and overtreatment emphasizes the need for accurate disease aggressiveness stratification. Owing to limitations of standard clinicopathological parameters, there is an unmet need for novel biomarkers that may assist clinicians in deciding therapeutic strategy. Although many studies have reported promising candidate biomarkers, few have stood the test of reproducibility and none has effectively reached clinical routine. Thus, based on our previous experience in epigenetic-based biomarkers and taking into consideration the 
Table 3

Multivariable analysis concerning disease-specific survival, disease-free survival, and progression-free survival

\begin{tabular}{|c|c|c|c|c|}
\hline & \multicolumn{2}{|l|}{ CAPRA risk score } & \multicolumn{2}{|l|}{ Prognostic grade groups ${ }^{\mathrm{a}}$} \\
\hline & Low CAPRA HR $(95 \% \mathrm{CI})$ & High CAPRA HR (95\% CI) & Groups 1-3 HR (95\% CI) & Groups $4-5$ HR $(95 \% \mathrm{CI})$ \\
\hline \multicolumn{5}{|c|}{ DSS (all patients) } \\
\hline \multicolumn{5}{|c|}{ Biomarker } \\
\hline \multicolumn{5}{|l|}{ Ki67 } \\
\hline$<$ P75 & 1 & 1 & 1 & 1 \\
\hline$\geq \mathrm{P} 75$ & $4.53(1.36-15.10)^{\mathrm{b}}$ & $1.40(0.74-2.69)$ & $2.13(0.44-10.20)$ & $2.18(1.09-4.38)^{\mathrm{b}}$ \\
\hline \multicolumn{5}{|l|}{$\mathrm{EZH} 2$} \\
\hline$<\mathrm{P} 75$ & 1 & 1 & 1 & 1 \\
\hline$\geq \mathrm{P} 75$ & $2.57(0.82-7.99)$ & $1.76(0.94-3.29)$ & $3.66(1.15-11.6)^{\mathrm{b}}$ & $0.72(0.37-1.40)$ \\
\hline \multicolumn{5}{|l|}{ SMYD3 } \\
\hline Weak & 1 & 1 & 1 & 1 \\
\hline Moderate & $2.19(0.27-17.86)$ & $1.86(0.64-5.38)$ & $1.55(0.32-7.44)$ & $0.50(0.14-1.79)$ \\
\hline Strong & $2.27(0.27-19.51)$ & $2.84(0.97-8.33)$ & $3.95(0.76-20.70)$ & $0.40(0.11-1.45)$ \\
\hline \multicolumn{5}{|c|}{ DFS (patients treated with curative intent) } \\
\hline \multicolumn{5}{|c|}{ Biomarker } \\
\hline \multicolumn{5}{|l|}{ Ki67 } \\
\hline$<\mathrm{P} 75$ & 1 & 1 & 1 & 1 \\
\hline$\geq \mathrm{P} 75$ & $9.20(1.27-66.44)^{\mathrm{b}}$ & $1.39(0.43-4.52)$ & $2.95(0.75-11.58)$ & $0.24(0.02-3.60)$ \\
\hline \multicolumn{5}{|l|}{$\mathrm{EZH} 2$} \\
\hline$<\mathrm{P} 75$ & 1 & 1 & 1 & 1 \\
\hline$\geq \mathrm{P} 75$ & $0.98(0.11-8.38)$ & $2.62(0.81-8.47)$ & $1.47(0.32-6.87)$ & $4.15(0.52-33.07)$ \\
\hline \multicolumn{5}{|l|}{ SMYD3 } \\
\hline Weak & 1 & 1 & - & - \\
\hline Moderate & $0.99(0.10-9.63)$ & $0.52(0.16-1.64)$ & $1^{\mathrm{c}}$ & $1^{\mathrm{c}}$ \\
\hline Strong & $2.44(0.22-27.15)$ & $0.91(0.29-2.85)$ & $3.04(0.96-9.62)$ & $0.18(0.01-2.65)$ \\
\hline \multicolumn{5}{|c|}{ PFS (ADT treated patients) } \\
\hline \multicolumn{5}{|c|}{ Biomarker } \\
\hline \multicolumn{5}{|l|}{ Ki67 } \\
\hline$<\mathrm{P} 75$ & 1 & 1 & 1 & 1 \\
\hline$\geq \mathrm{P} 75$ & $2.97(1.05-8.43)^{\mathrm{b}}$ & $1.12(0.64-1.97)$ & $1.22(0.36-4.16)$ & $1.29(0.71-2.32)$ \\
\hline \multicolumn{5}{|l|}{$\mathrm{EZH} 2$} \\
\hline$<$ P75 & 1 & 1 & 1 & 1 \\
\hline$\geq \mathrm{P} 75$ & $1.03(0.43-2.47)$ & $1.09(0.64-1.84)$ & $2.24(0.81-6.17)$ & $0.66(0.38-1.15)$ \\
\hline \multicolumn{5}{|l|}{ SMYD3 } \\
\hline Weak & 1 & 1 & 1 & 1 \\
\hline Moderate & $2.97(0.37-23.60)$ & $0.95(0.44-2.05)$ & $1.86(0.62-5.57)$ & $0.52(0.19-1.40)$ \\
\hline Strong & $2.47(0.31-19.79)$ & $0.85(0.38-1.92)$ & $1.94(0.56-6.69)$ & $0.40(0.15-1.09)$ \\
\hline
\end{tabular}

ADT = androgen deprivation therapy; DFS = disease-free survival; DSS = disease-specific survival; PFS = progression-free survival.

${ }^{a}$ Adjusted for patient age and clinical stage.

${ }^{\mathrm{b}}$ Values with statistical significance.

${ }^{\mathrm{c}}$ Reference category: SMYD3 weak/moderate.

most promising and scrutinized candidates [10-21], we sought to investigate, in a diagnostic prostate biopsy cohort, whether immunoexpression assessment, assisted by digital imaging analysis, might provide prognostically relevant information for patients with $\mathrm{PCa}$, discriminating indolent from aggressive disease, in a pretherapeutic setting.

Our study is based on a series of 189 consecutive diagnostic prostate biopsies from a single tertiary hospital, encompassing the full spectrum of clinically managed $\mathrm{PCa}$, as denoted by the different treatment strategies depicted. Interestingly, only a small proportion of patients were submitted to RP, which has been used as the main setting for validation of PCa biomarkers [10-12]. Thus, a prostate biopsy cohort broadens the biological and clinical features of PCa under evaluation, although at the cost of increased heterogeneity. Nevertheless, CS and GG allowed for statistically significant stratification of patients concerning DSS, validating the series, and notwithstanding the relatively limited size, our study further demonstrates the clinical value of GG, irrespective of treatment strategy, thus complementing previous reports [8,9] as it includes, for the first time, patients submitted to androgen deprivation therapy (ADT).

We found that PCa cases with high Ki67 expression displayed significantly worse DSS, both in univariable and multivariable analysis, in line with some previous findings in RP and prostate biopsy series [10-14]. Remarkably, Ki67 was the only tested biomarker that significantly associated with disease recurrence and progression, both in low/ intermediate risk CAPRA score groups. Thus, quantitative Ki67 immunoexpression adds to existing PCa prognostication tools, providing independent information, in a 
Table 4

Performance of various cutoffs mentioned in literature for Ki67 percentage nuclear expression in our cohort

\begin{tabular}{|c|c|c|c|c|c|}
\hline Study & $N$ & Date of diagnosis & $\begin{array}{l}\text { Median follow-up } \\
\text { (months) }\end{array}$ & Ki67 cutoff, \% & $\operatorname{HR}(95 \% \mathrm{CI})$ \\
\hline \multicolumn{6}{|c|}{ DSS_univariable analysis } \\
\hline Stattin et al. ${ }^{\mathrm{a}}[25]$ & 125 & $1975-1983$ & 71 & 3 & $1.27(0.73-2.21)$ \\
\hline Pollack et al. ${ }^{\mathrm{b}}[31]$ & 106 & $1987-1993$ & 62 & 3.5 & $1.33(0.76-2.34)$ \\
\hline Li et al. ${ }^{\mathrm{a}, \mathrm{b}}[32]$ & 108 & 1997-2003 & 72 & & \\
\hline Miyake et al. ${ }^{\mathrm{f}}[30]$ & 193 & $1997-2003$ & 63 & 5 & $1.77(1.001-3.14)$ \\
\hline Berney et al. ${ }^{a}$ [29] & 693 & 1990-1996 & 117 & & \\
\hline Gunia et al. ${ }^{\mathrm{f}}[28]$ & 528 & $1996-2003$ & 46.4 & & \\
\hline Rubio et al. ${ }^{\mathrm{b}, \mathrm{f}}[27]$ & 91 & 1997-2001 & 46.5 & & \\
\hline Bubendorf et al. ${ }^{\mathrm{f}}[24]$ & 137 & $1978-1993$ & 64.8 & 7.5 & $1.83(0.96-3.48)$ \\
\hline Zellweger et al. ${ }^{\mathrm{b}}[12]$ & 279 & 1996-2005 & 16 & 10 & $2.32(1.16-4.65)^{\mathrm{c}}$ \\
\hline Vis et $^{\text {al. }}{ }^{\mathrm{f}}[26]$ & 92 & $1980-1988$ & 112.8 & & \\
\hline Lobo et al. & 189 & 1997-2001 & 206 & 5.33 & $1.86(1.05-3.29)^{\mathrm{c}}$ \\
\hline \multicolumn{6}{|c|}{ DSS_multivariable analysis ${ }^{\mathrm{d}}$} \\
\hline Stattin et al. ${ }^{\mathrm{a}}[25]$ & 125 & $1975-1983$ & 71 & 3 & $1.24(0.67-2.29)$ \\
\hline Pollack et al. ${ }^{\mathrm{b}}[31]$ & 106 & $1987-1993$ & 62 & 3.5 & $1.28(0.69-2.38)$ \\
\hline Li et al. ${ }^{\mathrm{a}, \mathrm{b}}[32]$ & 108 & $1997-2003$ & 72 & & \\
\hline Miyake et al. ${ }^{f}[30]$ & 193 & $1997-2003$ & 63 & 5 & $1.39(0.74-2.59)$ \\
\hline Berney et al. ${ }^{a}$ [29] & 693 & 1990-1996 & 117 & & \\
\hline Gunia et al. ${ }^{\mathrm{f}}[28]$ & 528 & 1996-2003 & 46.4 & & \\
\hline Rubio et al. ${ }^{\mathrm{b}, \mathrm{f}}[27]$ & 91 & $1997-2001$ & 46.5 & & \\
\hline Bubendorf et al. $^{\mathrm{f}}$ [24] & 137 & $1978-1993$ & 64.8 & 7.5 & $2.00(0.997-3.99)$ \\
\hline Zellweger et al. $^{\mathrm{b}}$ [12] & 279 & 1996-2005 & 16 & 10 & $1.88(0.91-3.89)$ \\
\hline Vis et al. ${ }^{\mathrm{f}}[26]$ & 92 & $1980-1988$ & 112.8 & & \\
\hline Lobo et al. & 189 & 1997-2001 & 206 & 5.33 & $1.91(1.01-3.57)^{\mathrm{c}}$ \\
\hline \multicolumn{6}{|c|}{ DSS_multivariable analysis ${ }^{\mathrm{e}}$} \\
\hline Stattin et al. ${ }^{\text {a }}[25]$ & 125 & $1975-1983$ & 71 & 3 & $1.23(0.70-2.14)$ \\
\hline Pollack et al. $^{\mathrm{b}}[31]$ & 106 & $1987-1993$ & 62 & 3.5 & $1.27(0.72-2.24)$ \\
\hline Li et al. ${ }^{\mathrm{a}, \mathrm{b}}[32]$ & 108 & 1997-2003 & 72 & & \\
\hline Miyake et al. ${ }^{\mathrm{f}}[30]$ & 193 & $1997-2003$ & 63 & 5 & $1.70(0.95-3.01)$ \\
\hline Berney et al. ${ }^{a}$ [29] & 693 & 1990-1996 & 117 & & \\
\hline Gunia et al. ${ }^{\mathrm{f}}[28]$ & 528 & 1996-2003 & 46.4 & & \\
\hline Rubio et al. ${ }^{b, f}[27]$ & 91 & $1997-2001$ & 46.5 & & \\
\hline Bubendorf et al. $^{\mathrm{f}}[24]$ & 137 & $1978-1993$ & 64.8 & 7.5 & $1.77(0.93-3.38)$ \\
\hline Zellweger et al. ${ }^{\mathrm{b}}[12]$ & 279 & 1996-2005 & 16 & 10 & $2.25(1.12-4.51)^{\mathrm{c}}$ \\
\hline Vis et al. ${ }^{\mathrm{f}}[26]$ & 92 & $1980-1988$ & 112.8 & & \\
\hline Lobo et al. & 189 & 1997-2001 & 206 & 5.33 & $1.78(1.01-3.16)^{\mathrm{c}}$ \\
\hline
\end{tabular}

DSS $=$ disease-specific survival.

${ }^{a}$ Biomarker analysis in transurethral resection specimens.

${ }^{\mathrm{b}}$ Biomarker analysis in biopsy specimens.

${ }^{\mathrm{c}}$ Values with statistical significance.

${ }^{\mathrm{d}}$ Adjusted for patient age, clinical stage, and grade groups.

${ }^{\mathrm{e}}$ Adjusted for CAPRA score.

${ }^{\mathrm{f}}$ Biomarker analysis in radical prostatectomy specimens.

subgroup of patients which are at risk to endure overtreatment. In this setting, Ki67 assessment might assist in discriminating indolent from more aggressive $\mathrm{PCa}$ among low-risk patients, eventually identifying those that will mostly benefit from therapeutic intervention. Interestingly, results of immunohistochemical analysis of a single marker of cell proliferation-Ki67-seem to compare well with those of a commercially available cell-cycle progression score (Prolaris), a molecular test that evaluates RNA signature of several cell-cycle progression genes $[33,34]$. If confirmed in larger and independent series, Ki67 immunoexpression might provide an easier and less expensive method for widespread risk stratification of patients with $\mathrm{PCa}$ at diagnosis.

Several previous studies have indicated Ki67 as a promising prognostic biomarker for PCa [12,24-32]. Nevertheless, there is considerable heterogeneity among studies, including different cutoffs (most standing in the 5\%-10\% interval), cohort types (mostly RP specimens), sample sizes and endpoints, precluding a definitive judgment and translation to the clinics. Hence, we sought to determine the prognostic performance of the various Ki67 cutoffs in our series, using DSS as endpoint. Remarkably, and in addition to our P75 cutoff (Ki67 index of 5.33\%), only the 10\% 
cutoff reported by Zellweger et al. [12] revealed significant association with worse DSS, both in univariable and multivariable analysis. Interestingly, this is one of the few studies based on a prostate biopsy cohort. It should also be emphasized that, in our study, a more accurate and potentially more reproducible method for Ki67 assessment was used (digital image immunoscorer) and the Ki67 cutoff was defined based on the statistical distribution of results (P75) instead of empirically assigned values.

Owing to our previous experience in $\mathrm{PCa}$ epigeneticbased biomarkers, immunoexpression of 3 histones' modifying enzymes-EZH2, LSD1, and SMYD3-was also assessed. Whereas LSD1, previously shown to associate with risk of disease recurrence [18], did not confirm its potential, high EZH2 and SMYD3 immunoexpression predicted PCa-related death, both in univariable and multivariable analysis adjusted for CAPRA score. Moreover, in lower GG, high EZH2 immunoexpression predicted worse DSS, adjusted for patients' age and CS. These results are in line with previous reports and significantly extend their reach as they were mostly based on RP cohorts $[15,16,20,21,35,36]$. Additionally, they are biologically sound, as EZH2 is part of the polycomb complex group 2 , regulating genes involved in development and cell cycle progression by interacting both with histone and nonhistone proteins, functioning either as a transcriptional activator or repressor $[20,21,35]$, whereas SMYD3 is a methyltransferase that upregulates the androgen receptor, being involved in transcriptional regulation, either repressing tumor suppressor genes or inducing oncogene expression $[15,16,36]$. Importantly, both EZH2 and SMYD3 constitute potential therapeutic targets in $\mathrm{PCa}$ and, thus, expression assessment might also be predictive of response to targeted therapy.

Major limitations of our study include its retrospective nature, that may potentially bias the results, cohort size, making subgroup analysis for disease-free survival and progression-free survival difficult, treatment heterogeneity and the use of cases dating from 1997 to 2001, implying that sextant biopsy was performed and therapy was different from that currently used. Nevertheless, only long follow-up time enables appropriate assessment of DSS, which is a major endpoint for any biomarker. Moreover, although we have presented data concerning OS, the potential impact of comorbidities was not assessed, as we mostly focused on DSS, disease-free and progression-free survival. Furthermore, despite treatment heterogeneity, this study is based in a series of patients that were consistently managed by the same multidisciplinary clinical team over the years, imparting homogeneity in decision-making strategy. The fact that both CS and GG clearly stratified patients with PCa further supports the validity of our findings. Importantly, this prostate biopsy cohort allowed for improved evaluation of prognostic biomarker performance, which has been seldom achieved, as it represents the entire spectrum of primary PCa. Finally, the use of a digital image analysis system to improve biomarker quantification and reduce evaluation subjectivity, should also be emphasized. We found this to be a practical and easily learned procedure, which took only about 5 minutes per biomarker in each case, and that may be incorporated into routine practice, increasing the consistency and reliability of pathological assessment.

\section{Conclusions}

High Ki67, EZH2, and SMYD3 immunoexpression independently predicted $\mathrm{PCa}$ patient outcome, adjusted for standard clinicopathological parameters, in a cohort of diagnostic biopsies. If incorporated into routine practice, this assessment might assist clinicians in discriminating indolent from aggressive $\mathrm{PCa}$, improving treatment selection. Nevertheless, owing to the limitations of this study [use of sextant biopsies, lack of multiparametric magnetic resonance imaging data, heterogeneity of therapeutic strategies with predominance of advanced-stage $\mathrm{PCa}$ treated with ADT], validation of our findings in independent series is required.

\section{Ethics approval}

This study was approved by institutional review board of IPO Porto (CES-IPOFG-EPE215/013).

\section{References}

[1] Ferlay J, Soerjomataram I, Dikshit R, Eser S, Mathers C, Rebelo M, et al. Cancer incidence and mortality worldwide: sources, methods and major patterns in GLOBOCAN 2012. Int J Cancer 2015;136: E359-86.

[2] Siegel RL, Miller KD, Jemal A. Cancer statistics, 2016. CA Cancer J Clin 2016;66:7-30.

[3] Howlader N, Noone AM, Krapcho M, editors. SEER cancer statistics review. Bethesda, MD: National Cancer Institute; 2013.

[4] Gleason DF. Classification of prostatic carcinomas. Cancer Chemother Rep 1966;50:125-8.

[5] Epstein JI. An update of the Gleason grading system. J Urol 2010;183:433-40.

[6] Pierorazio PM, Walsh PC, Partin AW, Epstein JI. Prognostic Gleason grade grouping: data based on the modified Gleason scoring system. BJU Int 2013;111:753-60.

[7] Moch HUT, Reuter V, (Eds) WHO classification of tumours of the urinary system and male genital organs, 4th ed Lyon: IARC; 2016.

[8] Epstein JI, Zelefsky MJ, Sjoberg DD, Nelson JB, Egevad L, MagiGalluzzi C, et al. A contemporary prostate cancer grading system: a validated alternative to the Gleason score. Eur Urol 2016;69:428-35.

[9] Berney DM, Beltran L, Fisher G, North BV, Greenberg D, Moller H, et al. Validation of a contemporary prostate cancer grading system using prostate cancer death as outcome. Br J Cancer 2016;114: 1078-83.

[10] Kristiansen G. Diagnostic and prognostic molecular biomarkers for prostate cancer. Histopathology 2012;60:125-41.

[11] Fisher G, Yang ZH, Kudahetti S, Moller H, Scardino P, Cuzick J, et al. Prognostic value of Ki-67 for prostate cancer death in a conservatively managed cohort. Br J Cancer 2013;108:271-7.

[12] Zellweger T, Gunther S, Zlobec I, Savic S, Sauter G, Moch H, et al. Tumour growth fraction measured by immunohistochemical staining 
of Ki67 is an independent prognostic factor in preoperative prostate biopsies with small-volume or low-grade prostate cancer. Int J Cancer 2009:124:2116-23.

[13] Green WJ, Ball G, Hulman G, Johnson C, Van Schalwyk G, Ratan HL, et al. KI67 and DLX2 predict increased risk of metastasis formation in prostate cancer-a targeted molecular approach. Br J Cancer 2016;115:236-42.

[14] Tretiakova MS, Wei W, Boyer HD, Newcomb LF, Hawley S, Auman H, et al. Prognostic value of Ki67 in localized prostate carcinoma: a multi-institutional study of $>1000$ prostatectomies. Prostate Cancer Prostatic Dis 2016;19:264-70.

[15] Vieira FQ, Costa-Pinheiro P, Almeida-Rios D, Graca I, Monteiro-Reis S, Simoes-Sousa S, et al. SMYD3 contributes to a more aggressive phenotype of prostate cancer and targets cyclin D2 through H4K20me3. Oncotarget 2015;6:13644-57.

[16] Vieira FQ, Costa-Pinheiro P, Ramalho-Carvalho J, Pereira A, Menezes FD, Antunes L, et al. Deregulated expression of selected histone methylases and demethylases in prostate carcinoma. Endocr Relat Cancer 2014;21:51-61.

[17] Etani T, Suzuki T, Naiki T, Naiki-Ito A, Ando R, Iida K, et al. NCL1, a highly selective lysine-specific demethylase 1 inhibitor, suppresses prostate cancer without adverse effect. Oncotarget 2015;6:2865-78.

[18] Kahl P, Gullotti L, Heukamp LC, Wolf S, Friedrichs N, Vorreuther R, et al. Androgen receptor coactivators lysine-specific histone demethylase 1 and four and a half LIM domain protein 2 predict risk of prostate cancer recurrence. Cancer Res 2006;66:11341-7.

[19] Laitinen S, Martikainen PM, Tolonen T, Isola J, Tammela TL, Visakorpi T. EZH2, Ki-67 and MCM7 are prognostic markers in prostatectomy treated patients. Int J Cancer 2008;122:595-602.

[20] Melling N, Thomsen E, Tsourlakis MC, Kluth M, Hube-Magg C, Minner S, et al. Overexpression of enhancer of zeste homolog 2 (EZH2) characterizes an aggressive subset of prostate cancers and predicts patient prognosis independently from pre- and postoperatively assessed clinicopathological parameters. Carcinogenesis 2015;36:1333-40.

[21] van Leenders GJ, Dukers D, Hessels D, van den Kieboom SW, Hulsbergen CA, Witjes JA, et al. Polycomb-group oncogenes EZH2, BMI1, and RING1 are overexpressed in prostate cancer with adverse pathologic and clinical features. Eur Urol 2007;52:455-63.

[22] Cooperberg MR, Broering JM, Carroll PR. Risk assessment for prostate cancer metastasis and mortality at the time of diagnosis. J Natl Cancer Inst 2009;101:878-87.

[23] Heidenreich A, Bastian PJ, Bellmunt J, Bolla M, Joniau S, van der Kwast T, et al. EAU guidelines on prostate cancer. Part II: treatment of advanced, relapsing, and castration-resistant prostate cancer. Eur Urol 2014;65:467-79.

[24] Bubendorf L, Sauter G, Moch H, Schmid HP, Gasser TC, Jordan P, et al. Ki67 labelling index: an independent predictor of progression in prostate cancer treated by radical prostatectomy. J Pathol 1996; $178: 437-41$.
[25] Stattin P, Damber JE, Karlberg L, Bergh A. Cell proliferation assessed by Ki-67 immunoreactivity on formalin fixed tissues is a predictive factor for survival in prostate cancer. J Urol 1997;157: $219-22$.

[26] Vis AN, Noordzij MA, Fitoz K, Wildhagen MF, Schroder FH, van der Kwast TH. Prognostic value of cell cycle proteins p27(kip1) and MIB-1, and the cell adhesion protein CD44s in surgically treated patients with prostate cancer. J Urol 2000;164:2156-61.

[27] Rubio J, Ramos D, Lopez-Guerrero JA, Iborra I, Collado A, Solsona E, et al. Immunohistochemical expression of Ki-67 antigen, cox-2 and Bax/Bcl-2 in prostate cancer; prognostic value in biopsies and radical prostatectomy specimens. Eur Urol 2005;48:745-51.

[28] Gunia S, Albrecht K, Koch S, Herrmann T, Ecke T, Loy V, et al. Ki67 staining index and neuroendocrine differentiation aggravate adverse prognostic parameters in prostate cancer and are characterized by negligible inter-observer variability. World J Urol 2008;26: 243-50.

[29] Berney DM, Gopalan A, Kudahetti S, Fisher G, Ambroisine L, Foster CS, et al. Ki-67 and outcome in clinically localised prostate cancer: analysis of conservatively treated prostate cancer patients from the Trans-Atlantic Prostate Group study. Br J Cancer 2009;100: 888-93.

[30] Miyake H, Muramaki M, Kurahashi T, Takenaka A, Fujisawa M. Expression of potential molecular markers in prostate cancer: correlation with clinicopathological outcomes in patients undergoing radical prostatectomy. Urol Oncol 2010;28:145-51.

[31] Pollack A, Cowen D, Troncoso P, Zagars GK, von Eschenbach AC, Meistrich ML, et al. Molecular markers of outcome after radiotherapy in patients with prostate carcinoma: Ki-67, bcl-2, bax, and bcl-x. Cancer 2003;97:1630-8.

[32] Li R, Heydon K, Hammond ME, Grignon DJ, Roach M 3rd, Wolkov HB, et al. Ki-67 staining index predicts distant metastasis and survival in locally advanced prostate cancer treated with radiotherapy: an analysis of patients in radiation therapy oncology group protocol 86-10. Clin Cancer Res 2004;10:4118-24.

[33] Cuzick J, Stone S, Fisher G, Yang ZH, North BV, Berney DM, et al. Validation of an RNA cell cycle progression score for predicting death from prostate cancer in a conservatively managed needle biopsy cohort. Br J Cancer 2015;113:382-9.

[34] Bishoff JT, Freedland SJ, Gerber L, Tennstedt P, Reid J, Welbourn W, et al. Prognostic utility of the cell cycle progression score generated from biopsy in men treated with prostatectomy. J Urol 2014;192:409-14.

[35] Matsika A, Srinivasan B, Day C, Mader SA, Kiernan DM, Broomfield A, et al. Cancer stem cell markers in prostate cancer: an immunohistochemical study of ALDH1, SOX2 and EZH2. Pathology 2015;47:622-8

[36] Liu C, Wang C, Wang K, Liu L, Shen Q, Yan K, et al. SMYD3 as an oncogenic driver in prostate cancer by stimulation of androgen receptor transcription. J Natl Cancer Inst 2013;105:1719-28. 